\title{
Lichtbasierter Sensor zur kontinuierlichen Bestimmung von Chlordioxid
}

\author{
Stephanie Holz', Anatoly Glukhovskoy ${ }^{2}$ \\ ${ }^{1}$ Dr. Küke GmbH, Schaumburger Straße 11, 30900 Wedemark, Deutschland \\ 2Institut für Mikroproduktionstechnik, Leibniz Universität Hannover, An der Universität 2, 30823 \\ Garbsen, Deutschland \\ holz@kueke.de
}

\begin{abstract}
Zusammenfassung
Im Rahmen des Projektes "Lichtbasierte Analytik zur Bestimmung der Konzentration von Chlordioxid" $\left(\mathrm{LAClO}_{2}\right)$ wurde ein neuartiger Sensor zur Überwachung der Aufbereitung von Brauch-, Prozess- und Trinkwasser entwickelt und hergestellt. Im Wesentlichen zeichnet sich der Sensor neben seiner hervorragenden Integrierbarkeit in die vorhandene Anlagentechnik und EDV Struktur, durch die hohe Breitbandtauglichkeit und Kompaktheit des Systems aus. Das patentierte photometrische Messsystem erlaubt eine sehr genaue $\mathrm{pH}$ - und temperaturunabhängige und somit besonders resiliente Erfassung der Chlordioxidkonzentration. Die praktische Anwendbarkeit wurde durch den Aufbau und den Einsatz eines Prototyps in einem Realsystem demonstriert.
\end{abstract}

Keywords: Miniaturisierung,optische Sensorik,Chlordioxidsensoren,Chlordioxid,Online Sensor

\section{Grundlage optische Sensoren}

Zu Beginn des Projektes wurde ein Lastenheft basierend auf dem Stand der Technik erstellt. Alle für den Chlordioxid-Sensor und die Vergleichsanalytik relevanten Parameter wurden recherchiert und mit dem aktuellen Stand abgeglichen. Das Lastenheft diente dazu, den Sensor entwicklungsbegleitend auf die Systemanforderungen zu prüfen. Als Vorgaben wurde der Wellenlängenbereich für die Bestimmung der Chlordioxid $\left(\mathrm{ClO}_{2}\right)$ Konzentration in Leitungswasser festgelegt. Dabei kann die spezifische Absorption der elektromagnetischen Strahlung von $\mathrm{ClO}_{2}$ genutzt werden.

Der signifikante Absorptionsbereich von $\mathrm{ClO}_{2}$ liegt im Wellenlängenbereich von 270 bis $470 \mathrm{~nm}$. Mindestens drei technische Komponenten sind essentiell für den Aufbau eines optischen Messsystems zur Bestimmung der $\mathrm{ClO}_{2}$ Konzentration (Abbildung 1):

1. Emissionsquelle für elektromagnetische Strahlung im signifikanten Absorptionsbereich

2. Geeigneter Sensor für die zu messende Wellenlänge
3. Wahl eines geeigneten Messaufbaus hinsichtlich optischer Eigenschaften wie Absorption, Transmission und Reflexion

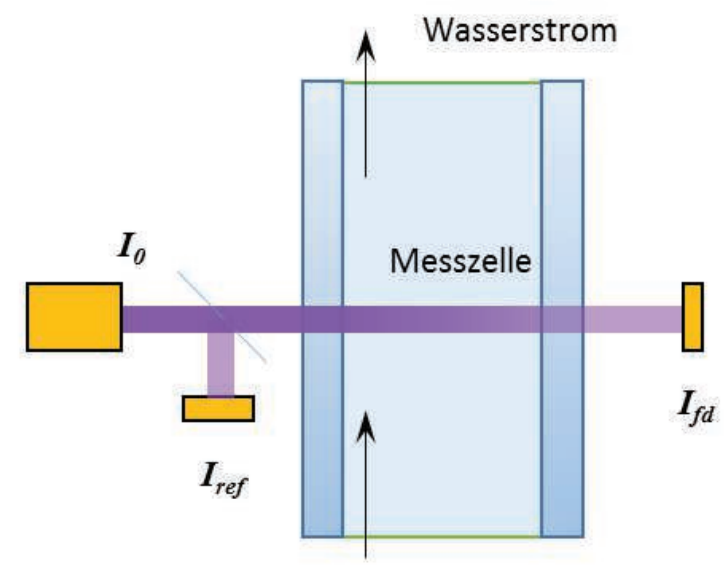

Abbildung 1: Schematische Darstellung der Einzelkomponenten (Messzelle, Lichtquelle, Fotodiode).

Aufgrund dessen, dass die meisten kommerziell erhältlichen Fotodioden in einem breiten Wellenlängenbereich sensitiv sind, ist die Verwendung von monochromatischer 
Strahlung, im konkreten die Verwendung einer Licht emittierenden Diode (LED), empfehlenswert. Zur Minimierung der photochemischen Dissoziation von $\mathrm{ClO}_{2}$, des Energieverbrauchs des Gesamtsystems und der Anpassung des Messsignals aufgrund des Dunkelstroms wird die LED im Pulsmodus betrieben (siehe Abbildung 2). Die zu betrachtenden Größen sind dabei: $I_{0}$ Laserstrahlintensität, $I_{\text {ref }}$ Laserstrahlintensität gemessen durch Referenz-Fotodiode, $I_{\mathrm{fd}}$ Signal der Mess-Fotodiode.

Transmission

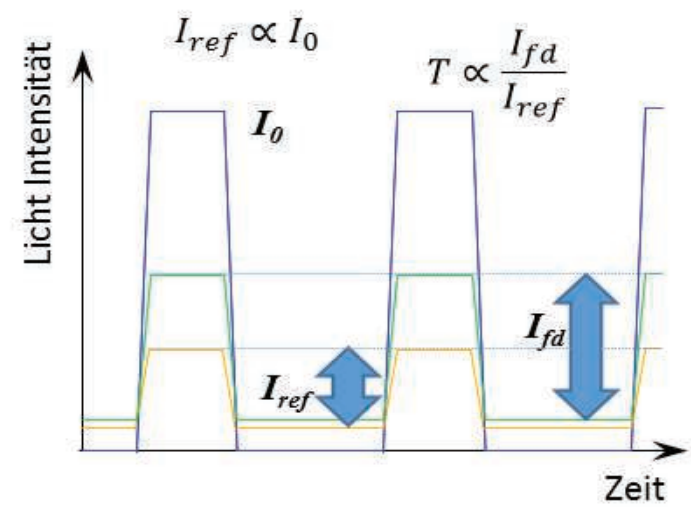

Abbildung 2: Signalform und Definition der Intensität des zugrundeliegenden Aufbaus.

\section{Entwicklung der Durchflusszelle}

Die Durchflusszelle dient der Medienführung mittels zwei Ein- und Ausstromanschlüssen sowie der gezielten Flussführung des Messvolumens über ein dünnkanaliges Radialsystem (siehe Strömungssimulation Abbildung 3). Der Medieneinstrom wird über die Unterseite der Durchflusszelle zugeführt, um Messsignal beeinträchtigende Lufteinschlüsse innerhalb der Zelle zu verhindern. Weiterhin ist die Durchflusszelle derart konstruiert, dass sowohl Justagemechanismen für das optische Teilsystem sowie das optische Fenster als auch die Klemmverbindung für die Dichtungen beinhaltet sind.

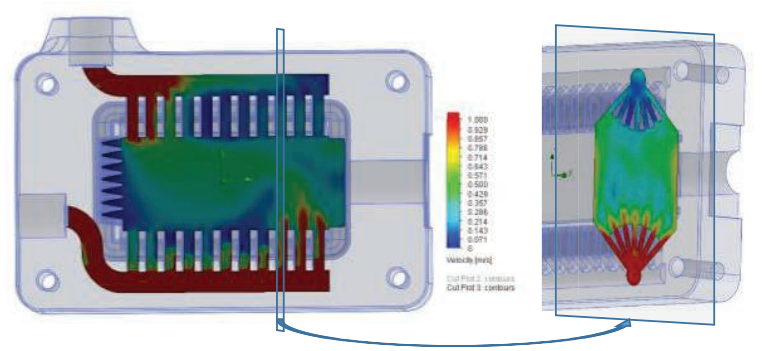
Abbildung 3:
Simulierte
(Fließgeschwindigkeit).

Die Durchflusszelle kann sowohl mit teiltransparenten Spiegeln als auch teiltransparenten optischen Fenstern bestückt werden. Der einfallende Lichtstrahl wird weiterhin über einen Winkel von $16^{\circ}$ durch ein optisches Fenster in die Durchflusszelle eingekoppelt (siehe Abbildung 4).

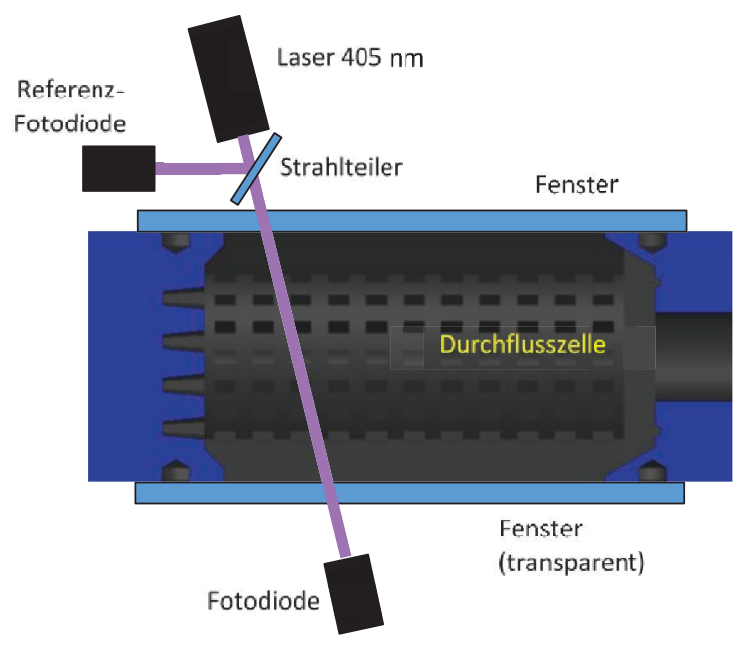

Abbildung 4: Schematischer optischer Versuchsaufbau.

Um den komplexen Aufbau des dünnwandigen Radialsystems zur Flussführung effizient und mit der notwendigen Präzision zu realisieren, wurde sich des Verfahrens des 3D-Druck aus dem Anwendungsbereich des Rapid Prototypings bedient. Die Oberflächen der Durchflusszelle wurden mittels eines 2-Komponenten Polydimethylsiloxans vakuumimprägniert. Die Ein- und Ausstromanschlüsse wurden mittels 2-Komponenten Epoxidklebers adhäsiv gebondet. Die optischen Komponenten wurden mechanisch mit der Durchflusszelle verbunden. Die Justierung des optischen Systems kann durch ein Befestigungssystem erfolgen.

\section{Optisches Fenster}

Als Grundmaterial für die optischen Fenster und Spiegel wird $2 \mathrm{~mm}$ starkes Quarzglas mit den Abmessungen von $30 \times 50 \mathrm{~mm}^{2}$ verwendet. Quarzglas weist gegenüber anderen Gläsern wie z.B. Borosilikatgläsern eine hohe Transmission von $95 \%$ im Wellenlängenbereich von 200 bis $450 \mathrm{~nm}$ auf. Quarzglas zeichnet sich weiterhin durch eine hohe mechanische Belastbarkeit sowie einen sehr geringen Wärmeausdehnungskoeffizienten aus. Vor Abscheidung der metallischen Oberflächen werden die Quarzglasrohlinge in erhitzter Piranha-Lösung (konzentrierte Schwefelsäure und $30 \%$ ige Wasserstoffperoxidlösung im Volumen- 
verhältnis 3:1) gereinigt, um organische Verschmutzung zu entfernen und die Oberfläche zu aktivieren. Um eine maximal mögliche Reflexion im relevanten Wellenlängenbereich zu erhalten, eignen sich im besonderen Aluminiumschichten. Die Aluminiumschicht wird mittels Elektronenstrahlverdampfens auf die Glasrohlinge abgeschieden. Es wurde verschiedene Schichtdicken der Aluminiumschicht abgeschieden, so dass die Anteile von transmittierter und reflektierter Strahlung durch die Schichtdicke bestimmt werden können. Die quantitative Bestimmung des Reflexions- und Transmissionsgrades der teiltransparenten optischen Komponenten wurde photometrisch ermittelt. Zur Bestimmung wurde ein Einfallswinkel von $16^{\circ}$ gewählt, so wie es im realen Betrieb ebenfalls der Fall ist. Die kontrollierbare Transmission erreicht bis zu $92 \%$ bei einem Reflexionsgrad von 8 bis $76 \%$ für Schichtdicken des Aluminiums von 0 bis $100 \mathrm{~nm}$. Obwohl Aluminium zur Selbstpassivierung neigt, kann eine Degradation der Aluminiumschicht beobachtet werden. Zur Vermeidung von Degradationserscheinungen wurde eine $250 \mathrm{~nm}$ dicke Siliziumdioxidschicht mittels PECVD auf die Aluminiumoberfläche abgeschieden.

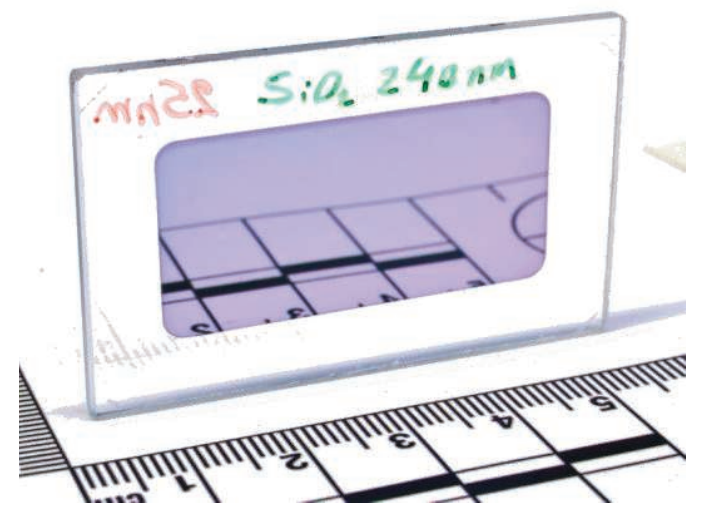

Bild 1: Fenster mit Reflexions- und Schutzschicht.

Um eine zusätzliche Abdichtung der optischen Fenster zu vermeiden, wurde ein Spannmechanismus am Außengehäuse der Durchflusszelle integriert, womit ein mechanisches Spannen der Fenster möglich ist. Zusätzlich wurden vorgeformte Silikondichtungsringe angefertigt, welche in die dafür vorgesehenen Kavitäten eingelassen werden können, und in Kombination mit der mechanischen Spannvorrichtung ein hermetisches Abdichten ermöglichen. Die Fensterrahmen werden mechanisch durch einen Schraubmechanismus an die Durchflusszelle gepresst. Nach vollständiger Evaluation der geeigneten optischen Fenster wurden diese mittels UV-aushärtendem Epoxidklebers an das Gehäuse adhäsiv gebondet. Die $2 \mathrm{~mm}$ starken Quarzfenster sind so ausgelegt, dass sie gegenüber einem Druck von 2 bar stabil sind, wobei ein Sicherheitsfaktor von 4 bereits einkalkuliert ist.

\section{Das optische Teilsystem}

Das optische Teilsystem beinhaltet neben den bereits erläuterten optischen Komponenten ebenso die LED, Fotodioden, Strahlteiler, Blenden, Fassung der optischen Komponenten und das Justagesystem.

Eine Herausforderung ist die Fokussierung des emittierten Lichtkegels, welcher durch eine Kollimationslinse gebündelt wird und der Halbleiterdiode, welche den interessanten Wellenlängenbereich emittiert. Die emittierte Wellenlänge liegt bei $405 \mathrm{~nm}$, wobei die Halbwertsbreite typenunabhängig in einem Bereich von $20 \mathrm{~nm}$ liegt. Der Extinktionskoeffizient für Wellenlängen im Bereich von $405 \mathrm{~nm}$ einer wässrigen Chlordioxidlösung liegt bei $600 \mathrm{~L} / \mathrm{mol} \cdot \mathrm{cm}$. Die zu erwartende Absorption ist um einen Faktor 2 geringer als im Vergleich zur maximalen Absorption bei $370 \mathrm{~nm}$. Die maximal mögliche Ausgangsleistung der Diode beträgt $45 \mathrm{~mW}$. Nichtsdestotrotz ist ein Betrieb unter $10 \mathrm{~mW}$ Ausgangsleistung aus Sicherheitsgründen zu empfehlen. Die Laserdiode wird in eine zylindrische $10 \mathrm{~mm}$ Fassung mit einer asphärischen Kollimationslinse platziert. Die SiliziumFotodiode hat eine Größe von 3,6 x 3,6 mm mit einer Messsignalgröße von 0,17 mA / mW für eine Wellenlänge von $405 \mathrm{~nm}$. Die Fotodioden werden ebenfalls in einer zylindrischen $10 \mathrm{~mm}$ Fassung platziert (siehe Abbildung 8). Der modulare Aufbau vereinfacht die Aufbaumechanismen und erhöht die Flexibilität des Systemaufbaus hinsichtlich einer Variation der optischen Komponenten.

Alle sensierenden und emittierenden Elemente können in die 3D gedruckte Fassung aufgenommen werden. Zusätzlich ist es möglich, den Strahlteiler sowie die konischen Blenden einzubetten. Sollte der Strahldurchmesser sich ausweiten, kommt es durch die konischen Blenden zu einer Reduzierung des Strahldurchmessers auf einen Durchmesser von $2 \mathrm{~mm}$ zur Fokussierung des Lichtstrahls auf das Zentrum der Fotodiode. Somit können Signalstörungen, welche mechanischen, thermischen oder hydraulischen Ursprungs sind, minimiert werden. Die Intensität der transmittierenden Strahlung wird mittels des Referenzstrahls 
normiert. Der Referenzstrahlteiler besteht aus einem $500 \mu \mathrm{m}$ dünnen reflektierenden Glasrohling und die referenzmessende Fotodiode ist in der entsprechenden Fassung fixiert. Die Fassung verhindert weiterhin die Möglichkeit, dass durch die Polarisationsrichtung des Lichtes erhöhte Intensitätsverluste auftreten, da ein falscher Einbau geometriebedingt nicht möglich ist.

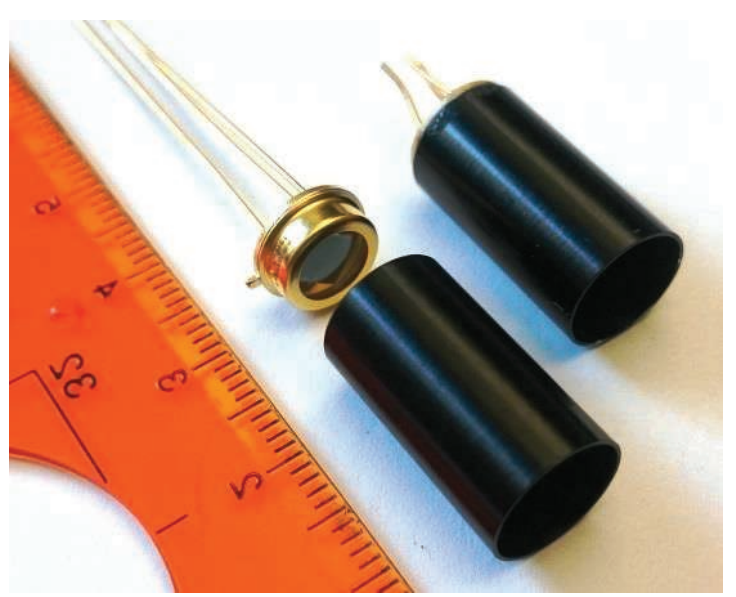

Abbildung 8: Beispielhaft ist das Fotodiodenmodul gezeigt.

\section{Messresultate}

Durch Aufzeichnung des Absorptionsspektrums des $\mathrm{ClO}_{2}$ ist die Bestimmung des Extinktionskoeffizienten für eine Wellenlänge von $405 \mathrm{~nm}$ möglich. Der Extinktionskoeffizient liegt bei $600 \mathrm{~L} / \mathrm{mol} \cdot \mathrm{cm}$. Der Messaufbau hat eine Länge von $24 \mathrm{~mm}$, so dass bei einem einfallenden Lichtstrahl mit einem Winkel von $16^{\circ}$ und unter Berücksichtigung des Brechungsindex von Wasser eine optische Weglänge von $25 \mathrm{~mm}$ erreicht wird. Mithilfe der Vereinfachung des Lambert-Beer Gesetzes wurde der Messbereich des Sensors bestimmt (blaues Parallelogramm).

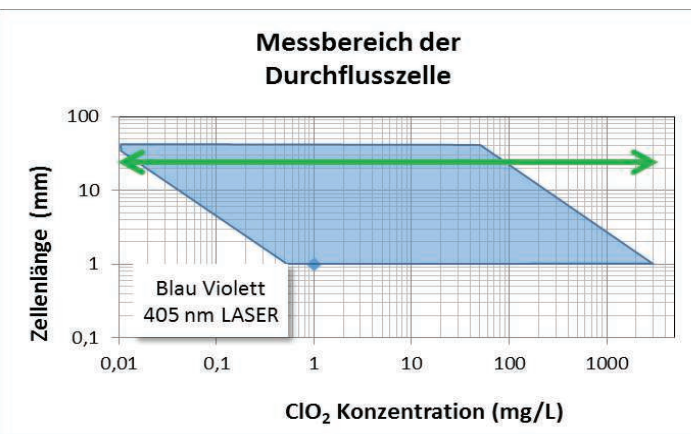

Abbildung 9: Theoretischer Messbereich des Sensors.
Basierend auf den ersten Messergebnissen konnte der Anpassungsfaktor der Transmission für das Gesamtsystem korrigiert werden. Im konkreten Fall beträgt der Extinktionskoeffizient $545 \mathrm{~L} / \mathrm{mol} \cdot \mathrm{cm}$ und die konzentrationsunabhängige Dämpfung liegt bei $0,04 / \mathrm{cm}$ (siehe Abbildung 10).

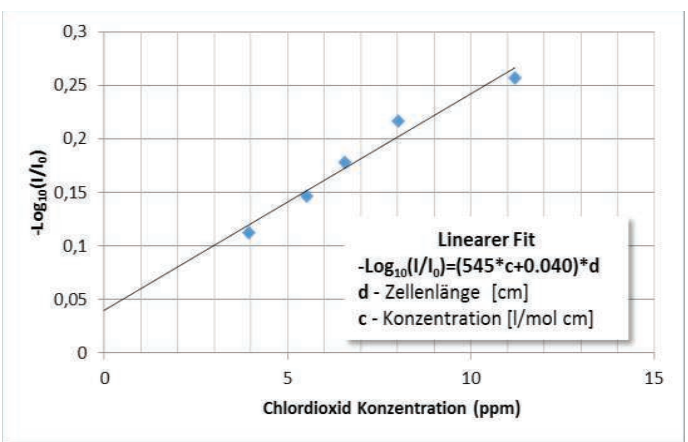

Abbildung 10: Darstellung der ermittelten Transmission - Konzentration Abhängigkeit.

\section{Referenzanalytik}

Die Referenzanalytik wurde erfolgreich recherchiert und erworben. Es wurde ein flexibler Aufbau für den Test-/Messstand gewählt. Der Durchmesser der Rohrleitung und das Material entsprechen einer Standard Kaltwasserleitung (KWL). Der Aufbau des Messstandes lässt sich in fünf Bereiche aufgeteilt: Zulauf, Durchflusskontrolle, Dosierstation, Sensorik und Ablauf.

Der Zulauf bestimmt das Medium, in welches die Dosierpumpen weitere Chemikalien einbringen. Dieser sieht für das Medium drei Grundversorgungen vor. Es ist möglich das System direkt mit VE-Wasser, Trinkwasser oder mit einem selbstdefinierten Medium aus einer Vorlage zu spülen und zu betreiben. Die Durchflusskontrolle reguliert den Wasserdruck und misst die Durchflussmenge mittels eines Schwebekörperdurchflussmessers.

Die Dosierstation bietet die Möglichkeit, über Pumpen gezielt das Medium zu verändern. Dies ermöglicht Versuche mit Säure-/BaseZugabe, Trübungsmittel, Farbstoffen etc.. Die Zudosierung des Chlordioxids kann ebenfalls über diese Pumpen erfolgen. Wichtig ist hierbei, dass die Zugabe durch Rückschlagventile gesichert ist, sodass keine Probelösung in die Pumpen gedrückt werden kann.

Die Sensorik besteht aus den exemplarisch gewählten Systemen zur Chlordioxidquantifizierung: DPD-Methode und 
amperometrische Messung, dem Prototypen des $\mathrm{LAClO}_{2}$-Projektes (direkt photometrisch), einer Durchflusszelle mit $\mathrm{pH}$ - und Redoxelektrode sowie einem Leitfähigkeitssensor. Die Zulaufschläuche der Durchflusszelle mit Elektroden sind über Adapter an die Kugelhähne der parallelen Zuläufe angeschlossen. Ebenso der $\mathrm{a}-\mathrm{LAClO}_{2}$, der finale Sensor des $\mathrm{LAClO}_{2}$-Projekts, wird über ein $1 / 2$ "-Gewinde eingebaut. Der Leitfähigkeitssensor ist über eine Gewindeverlängerung in ein T-Stück eingeschraubt, wobei angenommen wird, dass die Leitfähigkeit nur geringen Schwankungen unterliegt und die Diffusion ausreicht, um einen repräsentatives Ergebnis zu erzielen. Der Ablauf bietet die Möglichkeit der Rückführung des Medium, also eines Kreislaufsystems, oder der Verwerfung. Ein weiterer Anschluss ist außerdem zur Probenahme vorgesehen.

\section{Charakterisierung Sensor-1}

Die Messungen wurden durch manuelle Ermittlung des Chlordioxidgehalts unterstützt. Die Testzyklen zeigten bereits stabile Messungen, die allerdings noch nicht das gewünschte Auflösungsvermögen erreichten. Im Folgenden wurde daraufhin das Auflösungsvermögen optimiert.

\section{Driftverhalten}

Bei den Untersuchungen zum Langzeitverhalten wurde ein Driften beobachtet. Trotz des kontinuierlichen Abzugs des Dunkelstromanteils beider Signale ist es schwierig, die gegenseitige Linearität beider Verstärker zu gewährleisten. Transmission und Absorption sind definiert als das Verhältnis von Referenzstrom Iref zum Messstrom Isign.

Bei dieser Art von Systemen ist stets mit einem Bias zu rechnen. Dabei handelt es sich um eine konstante Größe, die das elektrische Signal überlagert und sich somit direkt auf das Messergebnis auswirkt. In diesem Fall erweitert sich die Gleichung zum Verhältnis $\left(I_{\text {rerf }}+a\right) /\left(I_{\text {sign }}+b\right)$, wobei $\boldsymbol{a}$ und $\boldsymbol{b}$ die Bias sind. Eine Änderung der Strahlintensität führt folglich zu einer Änderung des Messwertes, auch wenn das Verhältnis von Referenzstrom zu Messstrom konstant ist.

Nichtsdestotrotz führt dieses zu einem anderen Anwendungsfall, sodass die Instabilität der Laserstrahlintensität als Messinstrument genutzt werden kann. Während der LangzeitNullinienmessung (Messdauer > 72 Stunden) werden die Messwerte für das Referenz- und das Messsignal aufgenommen. Abbildung 10 zeigt den Zusammenhang der beiden Messgrößen. Trotz des Signalrauschens lässt sich die Abhängigkeit linear approximieren, was zu den beiden Näherungskoeffizienten führt. Sobald die Werte definiert sind, werden sie mit dem Auswertungsalgorithmus kompensiert, was zur Unterdrückung des Langzeitdrifts führt.

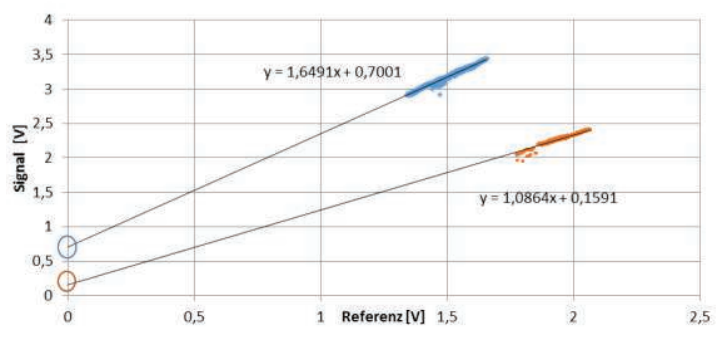

Abbildung 10: Beispielhaft dargestellter Biaseffekt.

\section{Komponenten-Design}

In Eigenfertigung werden sämtliche Kunststoffund Metallteile sowie die Glasbeschichtung als auch die Elektronik aufgebaut bzw. hergestellt. Nach der Optimierung des Designs, wird die Serienproduktion angestrebt. Dafür sind bereits entsprechende Anschlussvarianten vorgesehen, die den Einsatz des Sensors flexibel und variabel entsprechend den Anforderungen des Kunden gestaltet.

Der Prototyp liegt als Muster vor. Die Entwicklung bietet die Grundlage, um eine Fertigungskette für die Serie identifizieren zu können.

\section{Referenzmessung}

Die Ergebnisse der Testreihen sind beispielhaft in Abbildung 11 aufgeführt. Betrachtet wird der Bereich für Konzentrationen unter 0,4 mg Chlordioxid / L.

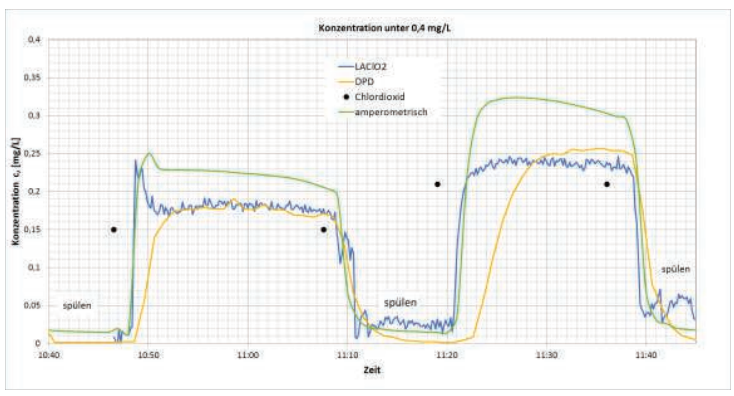

Abbildung 11: Messung am Teststand, verglichen wird der neue Sensor mit marktüblichen Systemen.

Am Teststand ist es möglich manuell Proben zu entnehmen und diese manuell auf Ihren Chlordioxidgehalt zu untersuchen. Die so 
bestimmten Werte dienen zum Abgleich mit den Messwerten der eingebauten Analytik. Im Graphen sind diese als schwarze Punkte notiert. Die Vergleichsmessungen wurden amperometrisch (Grün) und kolorimetrisch (DPD, Orange) durchgeführt. Die Messdaten des $\mathrm{LAClO}_{2}$ (Blau) zeigen eine gute Übereinstimmung mit der manuellen Referenz. Es soll an dieser Stelle nicht auf die Vergleichsinstrumente eingegangen werden, da diese typische Ergebnisse für ihre Funktionsart zeigen. Entscheidend ist der Nachweis, dass der Prototyp konkurrenzfähige Messergebnisse liefert.

Von besonderem Interesse sind die Ergebnisse für Konzentrationsmessungen im Bereich unter 0,5 mg Chlordioxid / L, da diese typischerweise im Bereich der Trinkwasserbehandlung auftreten und überwacht werden müssen. Die Messdaten zeigen noch ein Rauschen, das allerdings eine höhere Genauigkeit als $0,05 \mathrm{mg} / \mathrm{L}$ aufweist. Dies ist der Minimalwert, der als Konzentration nachgewiesen werden muss.

\section{Schlussfolgerung}

Die Funktionsweise des Systems wurde nachgewiesen. Durch das gewählte Design ist der variable Einbau als Online-Messsystem möglich. Die Umsetzung in die Serie soll in den nächsten Jahren abgeschlossen sein. 